\title{
Expression of Hyaluronidase-4 in a Rat Spinal Cord Hemisection Model
}

\author{
Yoshiyuki Tachi ${ }^{1}$, Tetsuhito Okuda ${ }^{1}$, Norio Kawahara ${ }^{1}$, Nobuo Kato ${ }^{2}$, \\ Yasuhito Ishigaki ${ }^{3}$, Tadami Matsumoto ${ }^{1}$ \\ ${ }^{1}$ Department of Orthopaedic Surgery, Kanazawa Medical University, Kahoku, Japan \\ ${ }^{2}$ Department of Physiology, Kanazawa Medical University, Kahoku, Japan \\ ${ }^{3}$ Medical Research Institute, Kanazawa Medical University, Kahoku, Japan
}

Study Design: Examination of hyaluronidase-4 (Hyal-4) expression in a rat spinal cord hemisection model.

Purpose: To determine the status of Hyal-4 expression after hemisection of the spinal cord, and the relationship between its expression and that of chondroitin sulfate proteoglycans (CSPGs).

Overview of Literature: CSPGs are expressed at the site of spinal cord injury and inhibit axon regeneration. Administration of exogenous chrondroitinase $\mathrm{ABC}(\mathrm{ChABC)}$, derived from bacteria, digested CSPGs and promoted axonal regrowth. Using a rat hemisection model, we have demonstrated peak CSPGs levels at by 3 weeks after injury but then decreased spontaneously. Could there be an endogenous enzyme similar to ChABC in the spinal cord? It has been suggested that Hyal-4 is involved in CSPG degradation.

Methods: A rat hemisection model was prepared and spinal cord frozen sections were prepared at 4 days and 1, 2, 3, 4, 5, and 6 weeks post-cordotomy and stained for CSPGs and Hyal-4 and subjected to Western blotting.

Results: CSPGs appeared at the injury site at 4 days after hemisection, reached a peak after 3 weeks, and then decreased. Hyal-4 was observed around the injury site from 4 days after cordotomy and increased until after 5-6 weeks. Double staining showed Hyal-4 around CSPGs. Western blotting identified a band corresponding to Hyal-4 from 4 days after hemisection.

Conclusions: Hyal-4 was expressed in a rat hemisection model in areas surrounding CSPGs, and as its peak was delayed compared with that of CSPGs. These results suggest the involvement of Hyal-4 in the digestion of CSPGs.

Keywords: Spinal cord injury; Chondroitin sulfate proteoglycans; Hyaluronidase

\section{Introduction}

Damage to the spinal cord induces progressive accumulation of reactive astrocytes around the lesion-induced hematoma within a few days [1], preceding the formation of a glial scar, within a few weeks [2]. Using a mouse spinal cord contusion model, Okada et al. [3] indicated that glial scar formation served to minimize spinal cord damage.
They demonstrated a lack of glial scar formation and an exacerbated damage in mice by knocking down "signal transducer and activator of transcription 3 ", a signal that induces the migration of reactive astrocytes [3]. However, various factors known to inhibit axonal regeneration are expressed in the glial scar and surrounding tissues [4]. First, reactive astrocytes inhibit axonal regeneration by expressing chondroitin sulfate proteoglycans (CSPGs)

Received Feb 19, 2014; Revised Apr 24, 2014; Accepted Apr 28, 2014

Corresponding author: Tetsuhito Okuda

Department of Orthopaedic Surgery, Kanazawa Medical University,

1-1 Daigaku, Uchinada, Kahoku, Ishikawa 920-0293, Japan

Tel: +81-76-286-2211, Fax: +81-76-286-4406, E-mail: okutetsu@kanazawa-med.ac.jp 
[5-8]. Second, in the white matter of the spinal cord surrounding the scar, myelin-associated glycoprotein, derived from myelin produced by oligodendrocytes, and Nogo-A are expressed $[9,10]$. Third, fibroblasts in the fibrous scar around the cavity express semaphorin $3 \mathrm{~A}$, which inhibits axonal regeneration $[11,12]$.

CSPGs, major components of the extracellular matrix, are expressed normally during development. They suppress axonal growth cones and control the direction of axonal extension $[13,14]$. CSPGs are degraded by chondroitinase $\mathrm{ABC}$ (ChABC), an enzyme derived from bacteria [15]. Administration of ChABC to a damaged spinal cord causes digestion of CSPGs expressed in glial scars, thereby enhancing axonal regrowth and functional recovery [16-19]. Furthermore, CSPGs expression is increased in a spinal cord hemisection model and then decreases [20], suggesting the enzymatic degradation of CSPGs.

The presence of endogenous enzymes digesting CSPGs is unknown except in bacteria. Recently, Kaneiwa et al. [21] reported that hyaluronidase-4 (Hyal-4), a human endogenous enzyme, specifically hydrolyses chondroitin sulfate (CS), suggesting its involvement in the digestion of CSPGs. In this study, we evaluated the presence or absence of Hyal-4 expression and the relationship between the decrease in CSPGs and Hyal-4 in a rat hemisection model.

\section{Materials and Methods}

The study protocol was conducted per the Guidelines for Animal Experiments of Kanazawa Medical University and was approved by the Animal Ethics Committee.

\section{Preparation of a rat spinal cord hemisection model}

Ten-week-old female Sprague Dawley rats ( $\mathrm{n}=42$, Sankyo Labo Service, Tokyo, Japan) were used. They were deeply anesthetized by intraperitoneal administration of pentobarbital sodium at $45 \mathrm{mg} / \mathrm{kg}$, and laminectomy of the 9th and 10th thoracic vertebrae was performed, and the 11th thoracic spinal cord was exposed. After incision of the dura matter, the right half of the spinal cord was sectioned with a razor blade. The spinal cord was hemisectioned beyond the median line to ensure the separation of half of the spinal cord. The rats were returned to their cages with free access to water and food without manual bladder expression.

\section{Preparation of anti-Hyal-4 antibody}

An antibody to Hyal-4 was ordered from Invitrogen (Life Technologies, Carlsbad, CA, USA). A segment considered unlikely to cross with other members of the hyaluronidase family was selected from the amino acid sequence of Hyal-4 (NP_001094250.1), and the amino acid sequence of the peptide to be synthesized was selected. The peptide sequence to be used for immunization was determined as CSYHIEASADREFVVKGRASD. The peptide was synthesized and rabbits were immunized with the peptide conjugated with keyhole limpet hemocyanin as the antigen. After confirming the increase in antibody levels, whole blood was sampled, and serum was obtained and the antibodies were purified with an affinity column.

\section{Immunofluorescence staining}

Rats were deeply anesthetized by intraperitoneal administration of pentobarbital sodium at $50 \mathrm{mg} / \mathrm{kg}$ at 4 days or $1,2,3,4,5$, or 6 weeks after hemisection, perfused with phosphate-buffered saline (PBS), and fixed by perfusion with $4 \%$ paraformaldehyde. A $16-\mathrm{mm}$ craniocaudal segment of the spinal cord containing the site of injury was excised and preserved overnight in $25 \%$ sucrose solution. The tissue was embedded in optimal cutting temperature compound (Sakura Finetech Japan, Tokyo, Japan), frozen, and sliced into $30-\mu \mathrm{m}$ thick coronal sections.

After the compound was washed off with PBS, the sections were treated with PBS containing 10\% normal goat serum $+0.3 \%$ Triton $\mathrm{X}-100$ at room temperature for 2 hours and allowed to react overnight with the primary antibody at $4^{\circ} \mathrm{C}$. The primary antibody, mouse monoclonal antibody CS56 (Sigma-Aldrich, St. Louis, MO, USA), was used at 1:200. The anti-Hyal-4 antibody was used after diluting with PBS containing 3\% normal goat serum $+0.3 \%$ Triton X-100 at 1:100. After washing the sections with PBS, they were allowed to react with the secondary antibody for 1 hour at room temperature. As the secondary antibody, Alexa488-conjugated goat anti-mouse IgG (1:200) and Alexa 594-conjugated goat anti-rabbit IgG (1:200) (Invitrogen, Life Technologies) were used after dilution with PBS containing 3\% normal goat serum $+0.3 \%$ Triton X-100. After washing the sections with PBS, they were mounted with Fluoromount (Cosmo Bio, Co., Ltd., Tokyo, Japan) and examined under a BZ-9000 (Keyence, Osaka, Japan), and images were captured. 


\section{Quantification of immunofluorescence stained-images}

Images obtained from three animals at each time point (total, 21 animals), with three slices per animal (total, 63 slices), were selected for analysis. The areas of CS56immunostained regions and anti-Hyal-4 antibodyimmunostained regions were determined in a $4.0 \times 4.0 \mathrm{~mm}$ square centered around the site of injury. Immunofluorescence staining for CS56 and anti-Hyal-4 antibody was measured with the software included with the BZ-9000, with the threshold set at $12(0-255)$. Non-specific areas such as the meninges and nerve roots were excluded from the measurements.

\section{Western blotting}

Animals were deeply anesthetized with pentobarbital at 4 days, 1, 2, 3, 4, 5, and 6 weeks after hemisection and perfused with PBS. A $10-\mathrm{mm}$ craniocaudal segment of the spinal cord centered around the site of injury was carefully and promptly resected after perfusion. Moreover, a 4-mm craniocaudal segment of the spinal cord centered around the site of injury was excised and homogenized with $200 \mu \mathrm{L}$ of lysis buffer (50 mM Tris- $\mathrm{HCl}$ [Wako Pure Chemical Industries, Ltd., Osaka, Japan]), $150 \mathrm{mM} \mathrm{NaCl}$ (Wako), and 1.0\% Nonidet P-40 (Nacalai Tesque Inc., Kyoto, Japan) in pure water, supplemented with protease inhibitor solution (prepared by dissolving EDTA-free complete mini [Roche, Ltd., Basel, Switzerland] at a rate of $1 \mu \mathrm{L} / 25 \mathrm{~mL})$. After centrifugation $(15,000 \mathrm{rpm}, 30$ minutes, $4^{\circ} \mathrm{C}$ ), $1.5 \mathrm{~mL}$ of the supernatant was separated as a cell lysis solution. An aliquot was diluted to $10 \mu \mathrm{L}$ by adding lysis buffer, mixed with the same volume of $2 \times$ SDS-PAGE sample buffer (mixture of Laemmli sample buffer [Bio-Rad Laboratories Inc., Hercules, CA, USA] and 2-mercaptoethanol [Sigma-Aldrich] at 19:1), and heat-treated at $95^{\circ} \mathrm{C}$ for 10 minutes. After heating, the sample was separated by SDS-PAGE, and transferred to a polyvinylidene difluoride membrane (Immunobilon, Millipore, Billerica, MA, USA) using a HrizBLOT apparatus (Atto, Tokyo, Japan). The membrane was first immersed in blocking solution (PBS-T solution containing 5\% skim milk) for 30 minutes. After incubation with the primary antibody (1,000-fold dilution of the prepared anti-Hyal-4 antibody) and the secondary antibody (10,000-fold dilution of goat anti-rabbit immunoglobulin/HRP, Dako, Tokyo, Japan), the membrane was washed twice, once with PBS-T for 5 minutes and then with $10 \mathrm{mM}$ PBS ( $\mathrm{pH}$ 7.4) for 5 minutes. For detection of bands, Immunostar (Wako) and Las-4000 (Fujifilm, Tokyo, Japan) were used.

\section{Evaluation of anti-Hyal-4 antibody}

Double-stranded DNA, in which restriction enzyme sites (5': BamHI, 3': XbaI) were added at the terminals of the base sequence of the full-length rat Hyal-4 gene (NM_001100780.1), was synthesized and subcloned into the BamHI-XbaI site of the vector (pBApo-CNV Pur). In the plasmid DNA obtained, the sequence of the synthetic gene was confirmed by single-strand analysis. The prepared plasmid was purified to transfection grade using the OIA Spin Mini prep kit (Qiagen, Hilden, Germany) (pBApo-CMV-rHyal4) and was transfected using Effecten (Qiagen) into HeLa cells, derived from a human uterine cervical cancer. The protein was extracted after 2-day culture, and Western blotting was carried out using antiHyal-4 antibody. pBApo-CMV (an empty vector) was used as a negative control.

\section{Statistical analysis}

Data are expressed as means \pm standard deviation. Analysis of variance was used to compare the quantified data of immunofluorescence staining for CS56 and anti-Hyal-4 antibody at each time point ( $n=9$, for each group), followed by the Bonferroni/Dunn post hoc test between points of measurement (StatView ver. 5.0, SAS Institute Inc., Cary, NC, USA). A p-value $<0.05$ was considered to indicate statistical significance.

\section{Results}

\section{Immunofluorescence staining}

1) Expression of CSPGs and Hyal-4

At 4 days after hemisection, weak staining for CS56 was observed at the site of injury (Fig. 1A), while weak staining with anti-Hyal-4 antibody was noted around the site of injury (Fig. 1H). After 2-3 weeks, strong staining for CS56 was noted in and at the margin of the site of injury (Fig. 1C, D), together with a gradual increase in the intensity of staining with anti-Hyal-4 antibody around the site of injury (Fig. 1J, K). However, at 4 weeks after hemisection and subsequently, the intensity of staining for CS56 
decreased gradually (Fig. 1E-G), while persistent staining with the anti-Hyal-4 antibody was noted (Fig. 1L-N). The area stained with CS56 measured $11.6 \pm 0.7 \times 10^{4} \mu \mathrm{m}^{2}$ at
4 days post-cordotomy, $15.5 \pm 1.1 \times 10^{4} \mu \mathrm{m}^{2}$ at 1 week, $20.6 \pm 1.7 \times 10^{4} \mu \mathrm{m}^{2}$ at 2 weeks, $25.0 \pm 2.6 \times 10^{4} \mu \mathrm{m}^{2}$ at 3 weeks, $12.8 \pm 1.5 \times 10^{4} \mu \mathrm{m}^{2}$ at 4 weeks, $8.7 \pm 1.0 \times 10^{4} \mu \mathrm{m}^{2}$ at
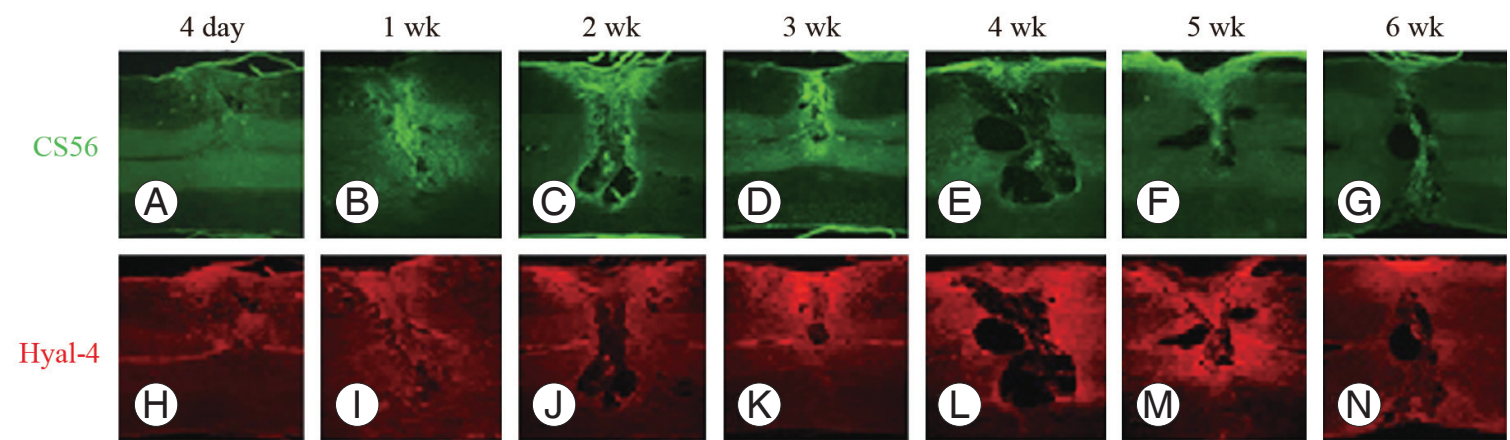

Merged
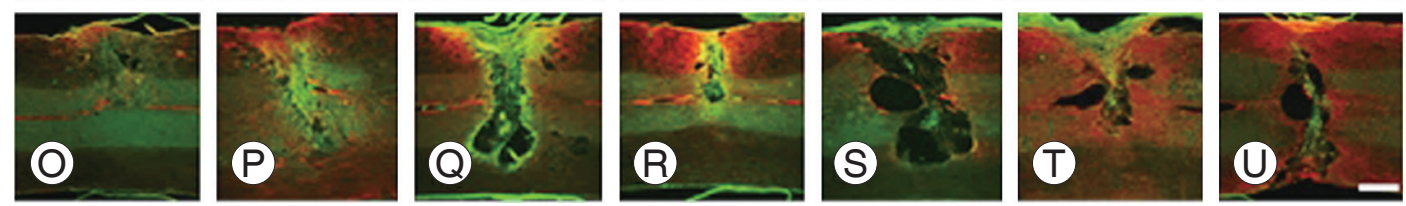

Fig. 1. Immunofluorescent images of chondroitin sulfate proteoglycans (CSPGs) and hyaluronidase-4 (Hyal-4) after spinal cord hemisection. Changes in immunological staining for CS56 (CSPGs) (green) and Hyal-4 (red) 4 days (A, H, 0), 1 week (B, I, P), 2 weeks (C, J, 0), 3 weeks (D, K, R), 4 weeks (E, L, S), 5 weeks (F, M, T), and 6 weeks (G, N, U) after spinal cord hemisection. Immunological staining for CS56 increased at the site of injury from 4 days to 3 weeks after hemisection (A-D) but then decreased (E-G). However, the Hyal-4-immunostained area increased around the site of injury from 4 days after hemisection and persisted for 4 weeks or longer $(\mathbf{H}-\mathbf{N})$. In double staining images, the Hyal-4-immunostained area (0-U) was observed around the CS56-stained area at the site of injury at all time points. In all images, the cranial side is to the left. Scale bar=500 $\mu \mathrm{m}$.

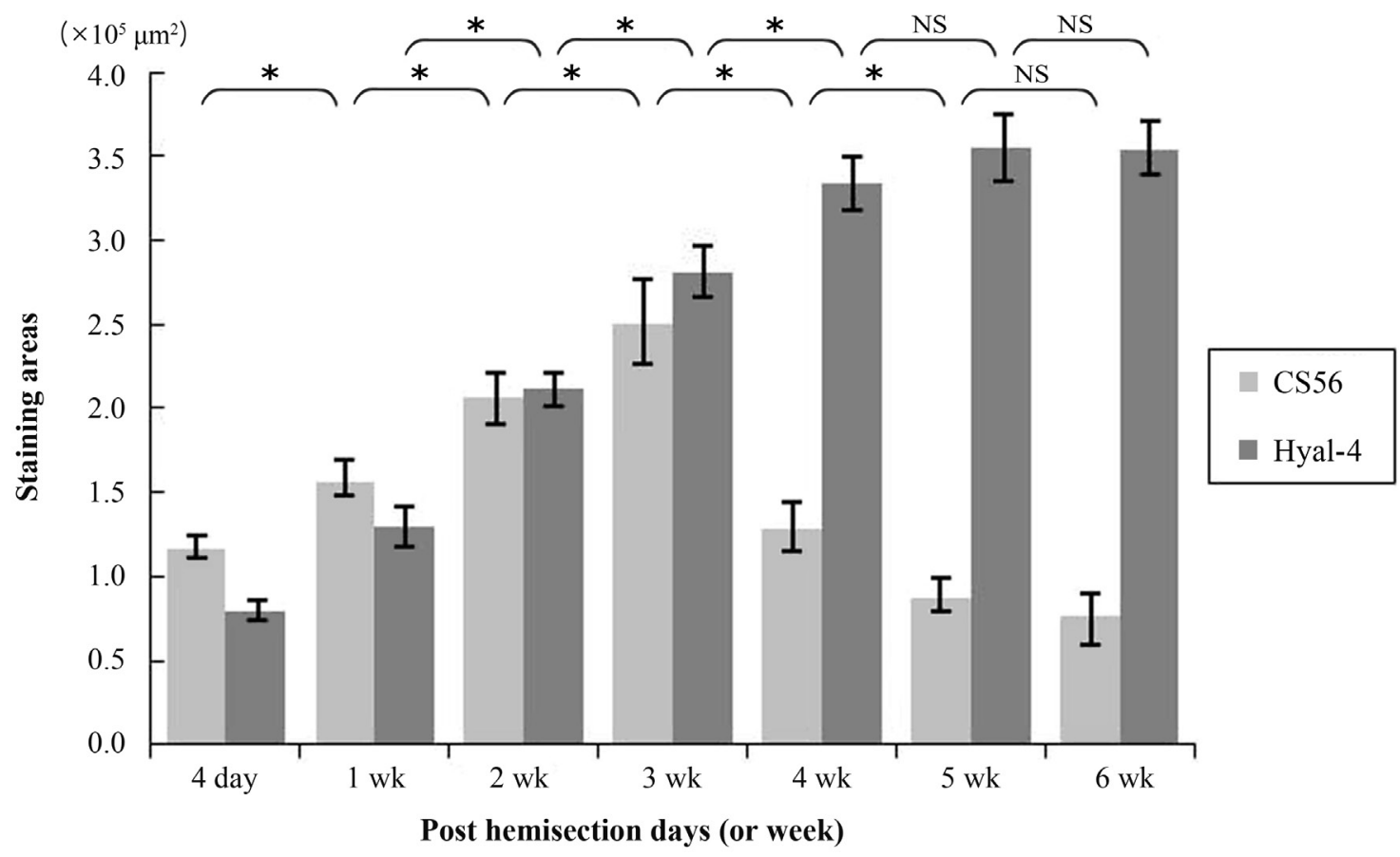

Fig. 2. Serial changes in the expression levels of chondroitin sulfate proteoglycans at the site of injury and hyaluronidase-4 (Hyal-4) around the site of injury. Data for the stained area are averaged over three slices obtained from three animals (total, 9 slices). Analysis of variance, using data at each time interval, showed significant differences in immunological staining for CS56 among various groups until 5 weeks after hemisection and staining for Hyal- 4 until 4 weeks after hemisection ( $\left.{ }^{*} p<0.0001\right)$. NS, not significant. 
5 weeks, and $7.7 \pm 1.4 \times 10^{4} \mu \mathrm{m}^{2}$ at 6 weeks. These results were consistent with the immunofluorescence staining. The Hyal-4-stained areas were $8.0 \pm 0.7 \times 10^{4}, 12.9 \pm 1.3 \times 10^{4}$, $21.3 \pm 1.0 \times 10^{4}, 28.0 \pm 1.5 \times 10^{4}, 33.4 \pm 1.7 \times 10^{4}, 35.6 \pm 2.0 \times 10^{4}$, and $35.4 \pm 1.7 \times 10^{4} \mu \mathrm{m}^{2}$, respectively (Fig. 2). Statistical analysis showed that the area stained for CS56 differed significantly among the measurement points, except after 5-6 weeks, increasing significantly from 4 days to 3 weeks after hemisection and decreasing significantly thereafter until 5 weeks after hemisection. The Hyal-4-stained area varied significantly among the measurement points until 4 weeks after, with significant increases from 4 days to 4 weeks post-cordotomy (Fig. 2).

\section{2) Relationship between CSPGs and Hyal-4}

The relationship between the decrease in CSPGs and Hyal-4 expression was evaluated using double staining. The intensity of staining for CS56 at the site of injury and for Hyal-4 around the site of injury increased gradually from 4 days to 3 weeks after cordotomy (Fig. 1O-R). Examination of the double-stained samples obtained 3 weeks after hemisection under high-power magnification showed that the areas stained for Hyal-4 and CS56 overlapped occasionally (Fig. 3). At 4 weeks after hemisection, staining for CS56 had diminished at the site of injury, whereas that for Hyal-4 had increased, compared with the pattern obtained at 3 weeks after hemisection (Fig. 1S). At 5-6 weeks, staining for CS56 diminished further, but that for Hyal-4 remained unchanged (Fig. 1T, U). Most of

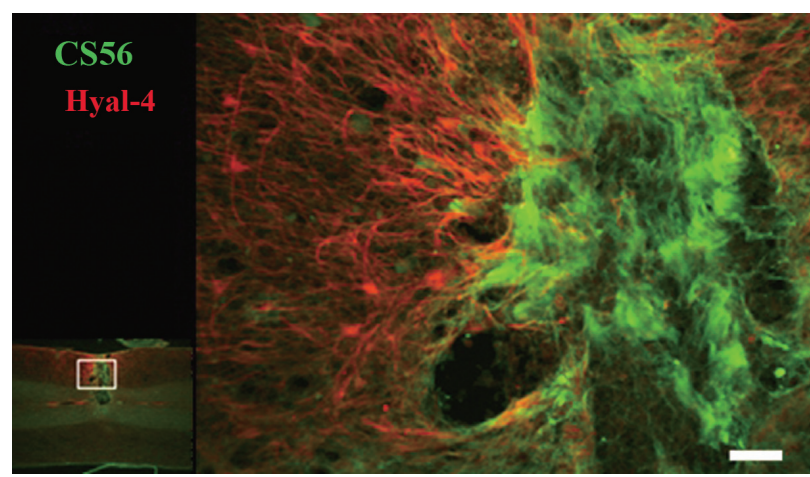

Fig. 3. High-magnification fluorescent staining images of chondroitin sulfate proteoglycans (CSPGs) and hyaluronidase-4 (Hyal-4) at 3 weeks after hemisection. Photomicrographs of the lesioned tissue 3 weeks after the hemisection. The results of immunofluorescence, stained for CS56 (CSPGs) (green) and Hyal-4 (red), are shown. The Hyal-4-immunostained area surrounds the CS56-positively stained area. In some parts, Hyal-4 staining invaded the area stained for CS56. Scale bar $=50 \mu \mathrm{m}$. the areas did not overlap at any point of evaluation and were clearly separated.

3) Characterization of rabbit antiserum against Hyal-4

Multiple protein bands were detected in samples obtained from the site of spinal cord injury at any time point (Fig. $4 \mathrm{~A})$. To evaluate the anti-Hyal-4 antibody, a plasmid
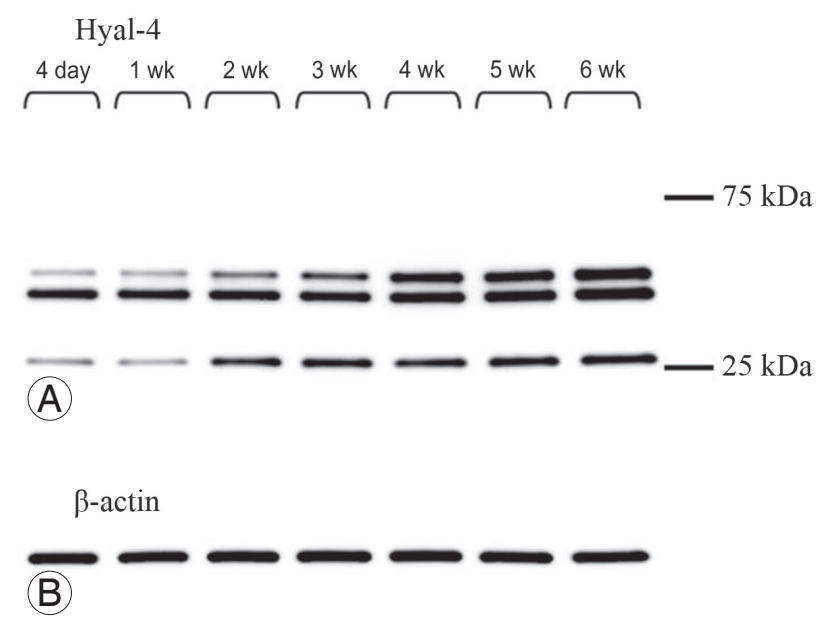

Fig. 4. Detection of hyaluronidase-4 (Hyal-4) expression after hemisection by Western blotting. Protein bands were detected at the same positions at each point of evaluation from 4 days to 6 weeks after hemisection (A). $\beta$-actin was used as a control for the protein level (B).

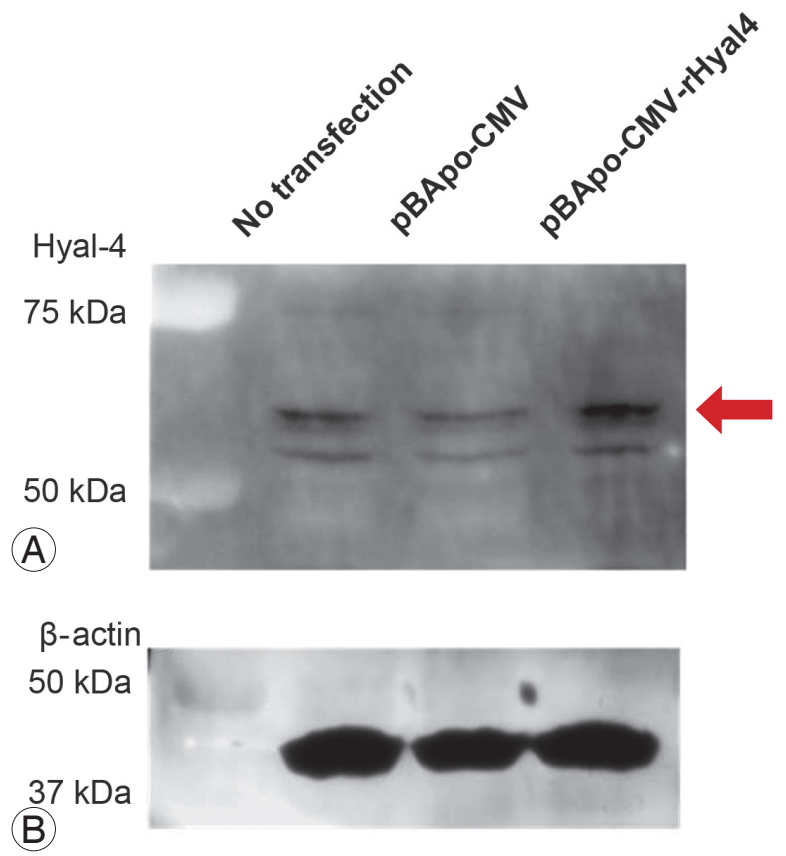

Fig. 5. Detection of hyaluronidase-4 (Hyal-4) expression. The expression of Hyal-4 protein in HeLa cells was examined by western blotting. A strong signal was noted at a position higher than $50 \mathrm{kDa}$ (indicated by arrow) (A). Results of analysis of the same lysates using $\beta$-actin as a control (B). 
vector expressing the full-length Hyal-4 rat gene was transfected into HeLa cells. Western blotting showed specifically dense protein bands at $50-75 \mathrm{kDa}$ in transfected cells (Fig. 5A). Also, the bands detected at $50-75 \mathrm{kDa}$ increased with time (Fig. 4A), supporting that these are derived from rat Hyal-4 gene (Figs. 4B, 5B).

\section{Discussion}

Hyal-4 evaluated in this study is one of the five members of the hyaluronidase family (hyaluronidase-1 [Hyal-1], Hyal-2, Hyal-3, Hyal-4, and Hyal-5) [22,23]. The hyaluronidase enzyme decomposes hyaluronic acid (HA) in various tissues [24]. Hyal-1 and Hyal-2 coordinately decompose high-molecular-weight HA [25]. HA is a GAG with a structure of alternating glucuronic acid and $\mathrm{N}$ acetyl glucosamine, resembling that of CS [25]. Kaneiwa et al. [21] demonstrated that Hyal-4 breaks the bond of galactosamine in CS and has a hydrolase activity specific to CS, but not HA, suggesting its involvement in the metabolism of CSPGs.

To examine Hyal-4 expression in the spinal cord, we prepared a new anti-Hyal-4 antibody in this study and used it for Western blotting. The anti-Hyal-4 antibody prepared was evaluated by transfecting HeLa cells with a vector expressing rat full-length Hyal-4 cDNA. A dense protein band of $50-75 \mathrm{kDa}$ was detected (Fig. 5A). Furthermore, while multiple bands were detected at the site of spinal cord injury (Fig. 4A), the results showed that the molecular weight of the band detected by anti-Hyal-4 antibody was higher than $50 \mathrm{kDa}$. These results indicated that the full-length Hyal-4 protein was located at the same position in samples extracted from rats and our antibody recognizes this polypeptide.

We also carried out an immunohistological evaluation of the rat hemisection model using CS56 as representative for CSPGs and anti-Hyal-4 antibody for Hyal-4. At the site of spinal cord injury, the increase in the expression of Hyal-4 after spinal cord injury was delayed compared with the increase in CSPGs, and the peak of Hyal4 was observed after the peak of CSPGs at 3 weeks postcordotomy and it then decreased. Additionally, Hyal4 was expressed in the area surrounding the tissue that expressed CSPGs at the site of spinal cord injury. Also, we observed occasional migration of the area expressing Hyal-4 into the CSPGs-expressing area, suggesting that Hyal-4 surrounding CSPGs was involved in the digestion of CSPGs. These findings are similar to those reported by Iseda et al. [20], who found GFAP-stained reactive astrocytes surrounding CSPGs in a rat spinal cord injury model. The results thus suggest that reactive astrocytes express Hyal-4. As mentioned above, reactive astrocytes also produce CSPGs [5-8], indicating that the same cells control the production and digestion of CSPGs. However, a limitation of this study is the lack of direct evidence from an experimental study using knock-out mice. Previous studies $[19,26]$ argued for a beneficial effect of ChABC on spinal cord injury or lesion, but the ChABC administered was applied exogenously and not of mammalian origin. In contrast, the present study revealed that CSPGs digestion could be achieved by endogenous expression of Hyal-4 in rats. Promotion of Hyal-4 expression would enhance digestion of CSPGs, and may open a new avenue to treatment of spinal cord injuries.

\section{Conclusions}

In a rat hemisection model, CSPGs were expressed at the site of injury and Hyal-4 was expressed in the surrounding area. The CSPG expression level decreased gradually from 3 weeks after hemisection, whereas Hyal4 increased gradually until 5-6 weeks after hemisection. Hyal-4 seems to be involved in digestion of CSPGs.

\section{Conflict of Interest}

No potential conflict of interest relevant to this article was reported.

\section{Acknowledgments}

This study was supported by a grant-in-aid from the AO Spine Japan.

\section{References}

1. Fitch MT, Doller C, Combs CK, Landreth GE, Silver J. Cellular and molecular mechanisms of glial scarring and progressive cavitation: in vivo and in vitro analysis of inflammation-induced secondary injury after CNS trauma. J Neurosci 1999;19:8182-98.

2. Fawcett JW, Asher RA. The glial scar and central nervous system repair. Brain Res Bull 1999;49:377-91.

3. Okada S, Nakamura M, Katoh H, et al. Conditional 
ablation of Stat3 or Socs3 discloses a dual role for reactive astrocytes after spinal cord injury. Nat Med 2006;12:829-34.

4. Silver J, Miller JH. Regeneration beyond the glial scar. Nat Rev Neurosci 2004;5:146-56.

5. McKeon RJ, Schreiber RC, Rudge JS, Silver J. Reduction of neurite outgrowth in a model of glial scarring following CNS injury is correlated with the expression of inhibitory molecules on reactive astrocytes. J Neurosci 1991;11:3398-411.

6. Haas CA, Rauch U, Thon N, Merten T, Deller T. Entorhinal cortex lesion in adult rats induces the expression of the neuronal chondroitin sulfate proteoglycan neurocan in reactive astrocytes. J Neurosci 1999;19:9953-63.

7. Asher RA, Morgenstern DA, Fidler PS, et al. Neurocan is upregulated in injured brain and in cytokinetreated astrocytes. J Neurosci 2000;20:2427-38.

8. McKeon RJ, Jurynec MJ, Buck CR. The chondroitin sulfate proteoglycans neurocan and phosphacan are expressed by reactive astrocytes in the chronic CNS glial scar. J Neurosci 1999;19:10778-88.

9. Chen MS, Huber AB, van der Haar ME, et al. Nogo$A$ is a myelin-associated neurite outgrowth inhibitor and an antigen for monoclonal antibody IN-1. Nature 2000;403:434-9.

10. Kim JE, Liu BP, Park JH, Strittmatter SM. Nogo-66 receptor prevents raphespinal and rubrospinal axon regeneration and limits functional recovery from spinal cord injury. Neuron 2004;44:439-51.

11. Pasterkamp RJ, Anderson PN, Verhaagen J. Peripheral nerve injury fails to induce growth of lesioned ascending dorsal column axons into spinal cord scar tissue expressing the axon repellent Semaphorin3A. Eur J Neurosci 2001;13:457-71.

12. Kaneko S, Iwanami A, Nakamura M, et al. A selective Sema3A inhibitor enhances regenerative responses and functional recovery of the injured spinal cord. Nat Med 2006;12:1380-9.

13. Brittis PA, Canning DR, Silver J. Chondroitin sulfate as a regulator of neuronal patterning in the retina. Science 1992;255:733-6.

14. Silver J, Edwards MA, Levitt P. Immunocytochemical demonstration of early appearing astroglial structures that form boundaries and pathways along axon tracts in the fetal brain. J Comp Neurol 1993;328:415-36.

15. Yamagata T, Saito H, Habuchi O, Suzuki S. Purifica- tion and properties of bacterial chondroitinases and chondrosulfatases. J Biol Chem 1968;243:1523-35.

16. Bradbury EJ, Moon LD, Popat RJ, et al. Chondroitinase $\mathrm{ABC}$ promotes functional recovery after spinal cord injury. Nature 2002;416:636-40.

17. McKeon RJ, Hoke A, Silver J. Injury-induced proteoglycans inhibit the potential for laminin-mediated axon growth on astrocytic scars. Exp Neurol 1995;136:32-43.

18. Yick LW, Cheung PT, So KF, Wu W. Axonal regeneration of Clarke's neurons beyond the spinal cord injury scar after treatment with chondroitinase ABC. Exp Neurol 2003;182:160-8.

19. Barritt AW, Davies M, Marchand F, et al. Chondroitinase $\mathrm{ABC}$ promotes sprouting of intact and injured spinal systems after spinal cord injury. J Neurosci 2006;26:10856-67.

20. Iseda T, Okuda T, Kane-Goldsmith N, et al. Single, high-dose intraspinal injection of chondroitinase reduces glycosaminoglycans in injured spinal cord and promotes corticospinal axonal regrowth after hemisection but not contusion. J Neurotrauma 2008;25:334-49.

21. Kaneiwa T, Mizumoto S, Sugahara K, Yamada S. Identification of human hyaluronidase-4 as a novel chondroitin sulfate hydrolase that preferentially cleaves the galactosaminidic linkage in the trisulfated tetrasaccharide sequence. Glycobiology 2010;20:300-9.

22. Csoka AB, Scherer SW, Stern R. Expression analysis of six paralogous human hyaluronidase genes clustered on chromosomes 3p21 and 7q31. Genomics 1999;60:356-61.

23. Csoka AB, Frost GI, Stern R. The six hyaluronidaselike genes in the human and mouse genomes. Matrix Biol 2001;20:499-508.

24. Meyer K, Chaffee E, Hobby GL, Dawson MH. Hyaluronidases of bacterial and animal origin. J Exp Med 1941;73:309-26.

25. Stern R. Devising a pathway for hyaluronan catabolism: are we there yet? Glycobiology 2003;13:105R$115 R$.

26. Ikegami T, Nakamura M, Yamane J, et al. Chondroitinase $\mathrm{ABC}$ combined with neural stem/progenitor cell transplantation enhances graft cell migration and outgrowth of growth-associated protein-43-positive fibers after rat spinal cord injury. Eur J Neurosci 2005;22:3036-46. 